\title{
MicroRNA-378 is involved in hedgehog- driven epithelial-to-mesenchymal transition in hepatocytes of regenerating liver
}

\author{
Jieun Kim', Jeongeun Hyun', Sihyung Wang ${ }^{1}$, Chanbin Lee ${ }^{1}$ and Youngmi Jung ${ }^{1,2}$
}

\begin{abstract}
Healthy livers have a remarkable regenerative capacity for reconstructing functional hepatic parenchyma after $70 \%$ partial hepatectomy (PH). Hepatocytes, usually quiescent in normal healthy livers, proliferate to compensate for hepatic loss after PH. However, the mechanism of hepatocyte involvement in liver regeneration remains unclear. Hedgehog $(\mathrm{Hh})$ pathway plays an important role in tissue reconstitution by regulating epithelial-to-mesenchymal transition (EMT) in liver disease. MicroRNA (miRNA) is involved in cell proliferation and differentiation during embryonic development and carcinogenesis. It was recently reported that miR-378 inhibits transdifferentiation of hepatic stellate cells into myofibroblasts by suppressing Gli-Krüppel family member 3 (Gli3), the Hh-target gene. We hypothesized that miR-378 influences EMT in hepatocytes by interfering with Hh signaling during liver regeneration. As hepatocytes were highly proliferative after PH in mice, miR-378 and epithelial marker, Ppar-g or E-cadherin were downregulated, whereas both Hh activators, Smoothened (Smo) and Gli3, and the EMT-inducing genes, Tgfb, Snail and Vimentin, were upregulated in the regenerating livers and in hepatocytes isolated from them. Compared to cells with or without scramble miRNA, primary hepatocytes transfected with miR-378 inhibitor contained higher levels of Gli3 with increased expression of the EMT-promoting genes, Tgfb, Snail, Col1a1, and Vimentin, suggesting that miR-378 influenced EMT in hepatocytes. Smo-depleted hepatocytes isolated from PH livers of Smo-flox mice showed downregulation of EMT-promoting genes and Gli3, with upregulation of miR-378 and E-cadherin compared to Smoexpressing hepatocytes from PH liver. In addition, delivery hepatocyte-specific AAV8 viral vector bearing Cre recombinase into Smo-flox mice impeded EMT in Smo-suppressed hepatocytes of PH liver, indicating that Smo is critical for regulating hepatocyte EMT. Furthermore, the application of miR-378 mimic into mice with PH delayed liver regeneration by interrupting hepatocyte EMT. In conclusion, our results demonstrate that miR-378 is involved in hepatocyte EMT by regulating Hh signaling during liver regeneration.
\end{abstract}

\section{Introduction}

The liver has remarkable regenerative ability, first described in Greek mythology and more recently proven in studies of rodents undergoing two-thirds partial hepatectomy $(\mathrm{PH})$. Liver regeneration after $\mathrm{PH}$ proceeds

\footnotetext{
Correspondence: Youngmi Jung (y.jung@pusan.ac.kr)

${ }^{1}$ Department of Integrated Biological Science, College of Natural Science,

Pusan National University, Pusan 46241, Korea

${ }^{2}$ Department of Biological Sciences, College of Natural Science, Pusan National

University, Pusan 46241, Korea

These authors contributed equally: Jieun Kim, Jeongeun Hyun

Edited by E. Candi
}

in an orderly fashion without necrosis and inflammation; therefore, murine models with surgical manipulation of $\mathrm{PH}$ are widely used for studying the biological processes of liver regeneration ${ }^{1}$. Surgical removal of two-thirds of the liver leads to a hyperplastic response in the remaining hepatic tissue until the original liver mass is reconstituted $^{2}$. Liver architecture and function are also retained during liver regeneration ${ }^{3}$. Mature hepatocytes, which are quiescent and rarely divide in normal healthy livers, reenter the cell cycle and replicate to compensate for the

\section{(c) The Author(s) 2018}

(c) (i) Open Access This article is licensed under a Creative Commons Attribution 4.0 International License, which permits use, sharing, adaptation, distribution and reproduction c. in any medium or format, as long as you give appropriate credit to the original author(s) and the source, provide a link to the Creative Commons license, and indicate if changes were made. The images or other third party material in this article are included in the article's Creative Commons license, unless indicated otherwise in a credit line to the material. If material is not included in the article's Creative Commons license and your intended use is not permitted by statutory regulation or exceeds the permitted use, you will need to obtain permission directly from the copyright holder. To view a copy of this license, visit http://creativecommons.org/licenses/by/4.0/. 
loss of liver mass ${ }^{2,4}$. Along with these hepatocytes, residual cholangiocytes and hepatic stellate cells (HSCs) are believed to mediate the repopulation of liver cells after $\mathrm{PH}^{5,6}$. However, it is poorly understood how the remnant liver regenerates the various types of liver cells.

Epithelial-to-mesenchymal transition (EMT) is a biological process in which epithelial cells gradually lose their adherence and apical-basal polarized phenotype, while gaining the migratory and invasive properties of mesenchymal cells ${ }^{7}$. There is altered expression of some epithelial (e.g. E-cadherin, Zona occludens-1, and Cytokeratin) and mesenchymal (e.g., Snail, Vimentin, $\alpha-$ Smooth muscle actin, and ColIa1) genes during this process $^{8}$. In chronically damaged livers, hepatocytes undergo EMT to escape destruction by TGFb, presenting the proliferative mesenchymal phenotype of myofibroblasts $(\mathrm{MFs})^{9}$. It was recently reported that hepatocytes have transiently decreased levels of hepatocyte nuclear factor 4 alpha, a marker of mature hepatocytes, as well as increased expression of EMT-promoting factors such as Yes-associated protein 1, and Gli-Krüppel family member 2 (Gli2), during the post-PH replicative period ${ }^{10}$. These findings indicate the transdifferentiation of hepatocytes during liver regeneration, but it is unclear how hepatocyte fate is regulated in this process.

Hedgehog (Hh) signaling is known to orchestrate tissue reconstruction and to be involved in EMT in damaged livers ${ }^{11-13}$. In chronically or severely injured livers, Hh signaling promotes the transition of quiescent HSCs and immature ductular cells into $\mathrm{MFs}^{13-16}$. Although mature hepatocytes in healthy adult livers are non-responsive to Hh signaling, hepatocytes in damaged livers activate $\mathrm{it}^{11,17}$. In particular, increased activation expression of the $\mathrm{Hh}$ ligands, Sonic $\mathrm{Hh}$ (Shh) and Indian $\mathrm{Hh}$, and the $\mathrm{Hh}-$ target genes, Gli1 and Gli2, is observed in the hepatocytes of regenerating livers after $70 \% \mathrm{PH}^{11}$. In $\mathrm{PH}$-treated mice with suppression of Hh signaling, liver regeneration is significantly inhibited, with reduced hepatocyte proliferation, decreased EMT, and disrupted matrix reconstitution, leading to reduced survival rates ${ }^{11}$. These findings suggest that $\mathrm{Hh}$ signaling is associated with proliferation and/or transdifferentiation of hepatocytes during post- $\mathrm{PH}$ liver regeneration.

MicroRNAs (miRNAs) have been implicated in cell proliferation and differentiation during embryonic development and cancer progression ${ }^{18,19}$. Several miRNAs, such as miR-101 and miR-181a, are reported to be involved in TGFb-induced EMT of hepatocytes in liver cirrhosis and hepatocellular carcinoma ${ }^{20,21}$. However, it remains unclear how and what kinds of miRNAs are involved in the hepatocyte reprogramming during normal liver regeneration. We recently demonstrated that miR-378 is involved in HSC transition in the fibrotic liver by interacting $\mathrm{Hh}$ signaling ${ }^{22}$. Given that $\mathrm{Hh}$ signaling is associated with the fate-change of hepatocytes and HSCs in the liver-repair process, it is possible that miR-378-mediating Hh signaling influences the EMT of hepatocytes in regenerating livers after PH. We investigated whether and how miR-378 is involved in hepatocyte transition during this process. We found that reduced miR-378 leads to the activation of $\mathrm{Hh}$ signaling which is involved in EMT in regenerating liver. Overexpression of miR378 impedes liver regeneration in mice by suppressing hepatocyte EMT. Taken together, these findings suggest miR-378 as an important regulator for hepatocyte transdifferentiation in regenerating livers.

\section{Results}

\section{Expressional changes of miR-378 with Hh-target genes in regenerating livers}

To generate the experimental model of adult normalliver regeneration, we performed $70 \% \mathrm{PH}$ on wild-type (WT) mice, who were sacrificed $1,3,6,12,24,48,72$, or $96 \mathrm{~h}$ post-surgery. The liver weight (LW) and LW-to-body weight (BW) ratio (LW/BW) began to be elevated at $48 \mathrm{~h}$, increasing by almost 2.3 -fold at $96 \mathrm{~h}$ compared with livers at $0 \mathrm{~h}$ post-PH (Supplementary Figure S1A). Histologic and enzymatic damage, assessed with hematoxylin and eosin (H\&E) staining and serum ALT/AST analysis, respectively, was observed in post-PH livers for $24 \mathrm{~h}$, but the injuries significantly recovered by $48 \mathrm{~h}$ post-PH (Supplementary Figures S1B\&C). In addition, immunostaining with Ki67, a marker of cell proliferation, showed the accumulation of Ki67-positive hepatocytes at $24 \mathrm{~h}$. The number of these cells sharply increased at $48 \mathrm{~h}$ and was maintained until $96 \mathrm{~h}$ (Supplementary Figure S2). These results confirmed that the murine $\mathrm{PH}$ model was successfully generated.

Since the EMT process occurs during liver regeneration after $\mathrm{PH}^{5}$, we analyzed the expression of EMT-related genes in liver tissues. These levels in the regenerating liver were compared with those in resected liver tissue at each post-PH time point. Expression of Tgfb, EMT-promoting factor $^{23,24}$, immediately increased $1 \mathrm{~h}$ after $\mathrm{PH}$ and declined to nearly basal levels at $6 \mathrm{~h}$. And then, Tgfb began to be upregulated at $48 \mathrm{~h}$, and then rapidly downregulated at $96 \mathrm{~h}$. The mRNA expression of Snail, TGFb-regulated transcription factors promoting $\mathrm{EMT}^{23}$, was immediately raised $1 \mathrm{~h}$ after $\mathrm{PH}$, peaking at almost 9-fold above basal levels at $6 \mathrm{~h}$ before being rapidly downregulated to nearly basal levels at $12 \mathrm{~h}$. Thereafter, Snail transiently increased and decreased, returning to basal levels at $96 \mathrm{~h}$. Expression of Vimentin, a mesenchymal marker, was maintained at the basal level until $12 \mathrm{~h}$, then increased significantly until $96 \mathrm{~h}$. Meanwhile, Ppar-g and E-cadherin, EMT inhibitor $^{25}$, were downregulated at $6 \mathrm{~h}$ at which point Snail expression was higher, and showed steadily low 

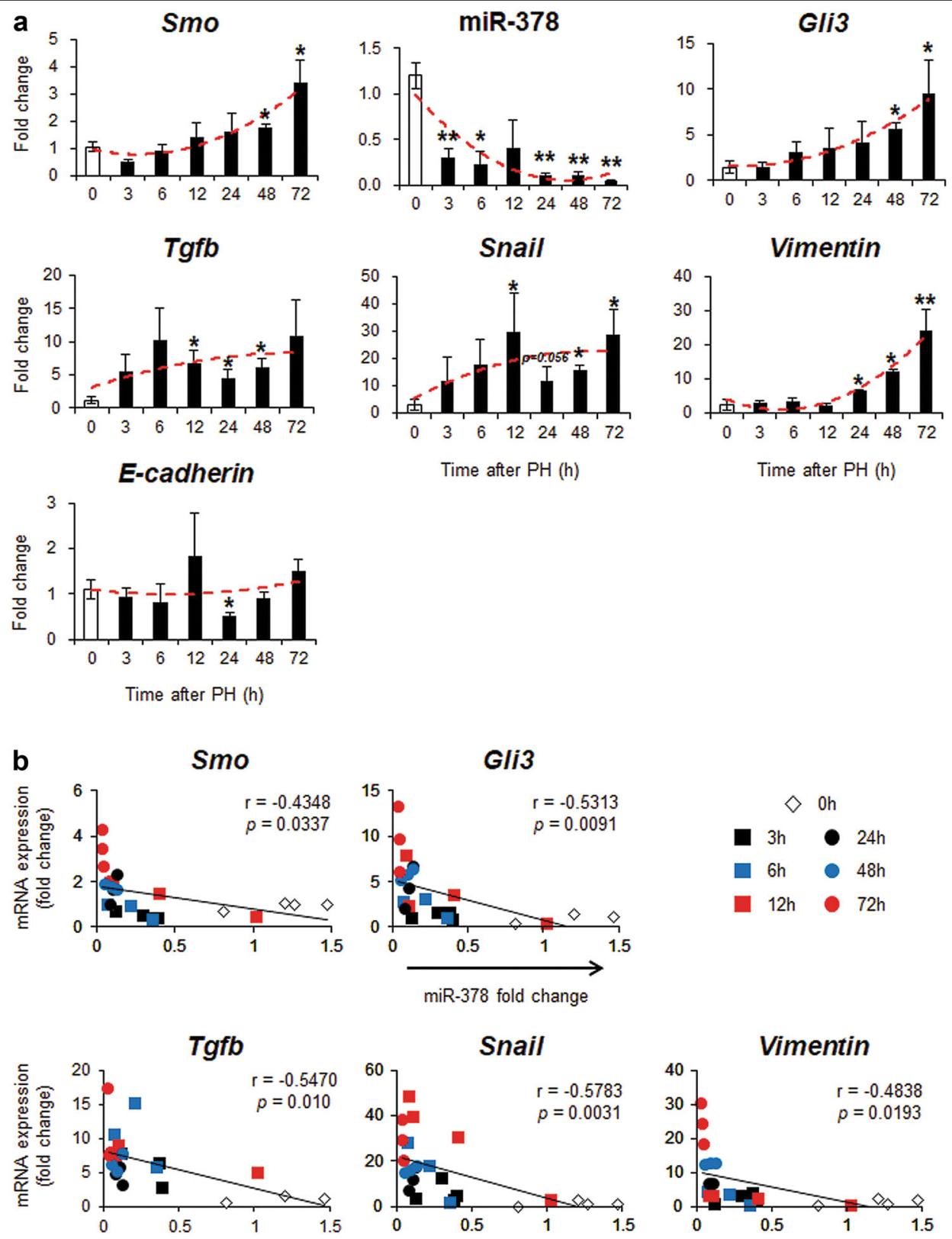

Fig. 1 Expressional changes of miR-378, Hh target genes, and EMT activator in hepatocytes from mice with PH. a Expression of Smo, miR378, Gli3, Tgfb, Snail, Vimentin, and E-cadherin was assessed by qRT-PCR analysis in primary hepatocytes isolated from mice at 3, 6, 12, 24, 48 and $72 \mathrm{~h}$ after $\mathrm{PH}$. All results of relative expression values are shown as mean \pm s.e.m. of triplicate experiments (unpaired two-sample Student's $t$ test, ${ }^{*} p<0.05$ ${ }^{* *} p<0.005$ vs. 0 h). b Pearson's correlation coefficient between miR-378 expression and Smo, Gli3, Tgfb, Snail, and Vimentin ( $N=24$, Pearson's correlation $r=-0.435, p=0.034$ with Smo; $r=-0.531, p=0.009$ with Gli3; $r=-0.547, p=0.01$ with Tgfb; $r=-0.578, p=0.003$ with Snail; $r=$ $-0.484, p=0.019$ with Vimentin)

levels until it was restored to nearly its basal level at $72 \mathrm{~h}$ (Supplementary Figure S3A).

Given that Hh signaling orchestrates EMT in the liverrepair process ${ }^{5,13}$ and that miR-378 targeting GLI3 influenced the transition of HSCs, with expression regulated by $\mathrm{SMO}^{22}$, we examined the expression of miR-378, Smo, and Gli3 in regenerating livers. Smo was slightly upregulated at $3 \mathrm{~h}$, rapidly downregulated at $6 \mathrm{~h}$, and then tended to increase gradually until $96 \mathrm{~h}$. MiR-378 inhibited by SMO revealed the opposite expression pattern with Smo. MiR378 showed lowered expression at $3 \mathrm{~h}$, then began to elevate, peaking at $12 \mathrm{~h}$, then gradually decreasing until $96 \mathrm{~h}$. Expression of Gli3 targeted by miR-378 greatly increased at $6 \mathrm{~h}$, similar to the pattern of Smo, and declined to below basal levels at $12 \mathrm{~h}$, the point at which miR-378 was highly expressed. It then gradually increased until $72 \mathrm{~h}$ 
(Supplementary Figure S3B). These findings indicate that EMT occurs in regenerating liver and SMO-GLI3-miR-378 axis is associated with this process.

\section{Hepatocytes underwent EMT in regenerating livers after $\mathrm{PH}$}

When hepatocytes are treated with specific triggers, such as TGFb, hepatitis $C$ viral protein, or organochlorine pesticides, they undergo an EMT/MET-like process ${ }^{24,26-29}$. Emerging evidence also supports a hepatocyte-mediated EMT-like process in human liver disease and murine liver fibrosis $^{30-32}$. Given that miR-378 influences GLI3 expression in the liver repair process ${ }^{22}$ and the expressional changes of EMT-related and Hh-target genes were observed in PH livers, we investigated whether hepatocytes undergo an EMT and whether miR-378-mediated Hh signaling contributes to this process. Hepatocytes were isolated from $\mathrm{PH}$ livers to evaluate hepatocyte-specific changes in gene expression during liver regeneration. Compared with primary hepatocytes isolated from quiescent livers $(0 \mathrm{~h})$,
Smo mRNA levels in hepatocytes from $\mathrm{PH}$ given mice were maintained at basal levels until $12 \mathrm{~h}$, then began to increase, showing significant upregulation at 48 and $72 \mathrm{~h}$. MiR-378 expression showed consistent reduction, below $40 \%$ of basal levels, whereas the expression of Gli3 targeted by miR-378 ${ }^{22}$ tended to increase gradually, with significant upregulation at 48 and $72 \mathrm{~h}$. Expression of $T g f b$ and Snail gradually increased and showed the plateau after presenting the significant elevation at $12 \mathrm{~h}$. Vimentin was upregulated at $24 \mathrm{~h}$, peaking at $72 \mathrm{~h}$, while E-cadherin was downregulated at $24 \mathrm{~h}$ and restored to nearly its basal level at $48 \mathrm{~h}$ (Fig. 1a). MiR-378 expression was inversely correlated with the Hh-target genes, Smo and Gli3, and the EMT-related genes, Tgfb, Snail and Vimentin, in hepatocytes from mice PH livers (Fig. 1b). SMO protein levels and the nuclear form of Gli3 were significantly elevated at $48 \mathrm{~h}$ and maximized at $72 \mathrm{~h}$, supplementary their RNA expression in hepatocytes. Because p65 was reported to be activated by Smo and to inhibit miR-378 transcription $^{22}$, p65 expression was examined. Expression
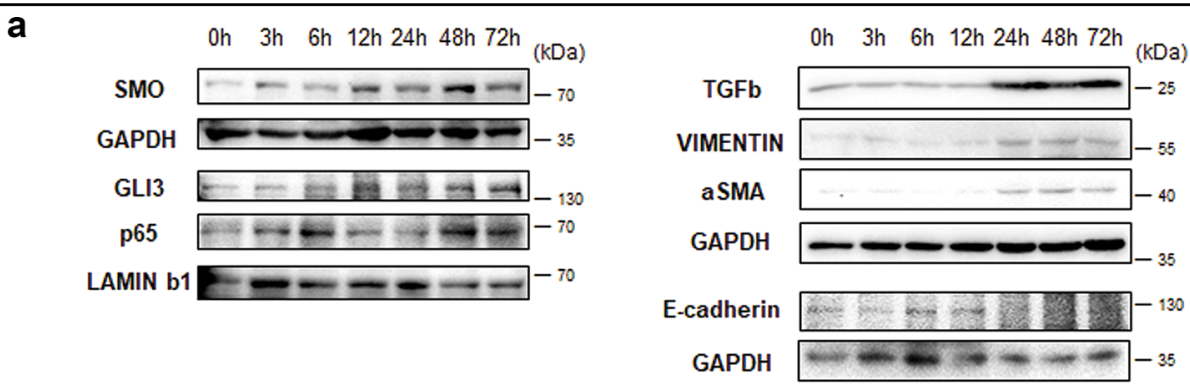

b

SMO

GLI3

p65
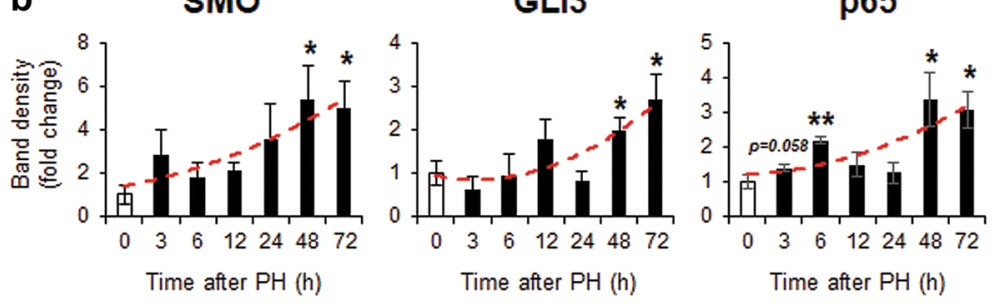

TGFb

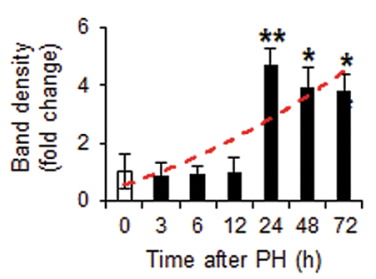

VIMENTIN

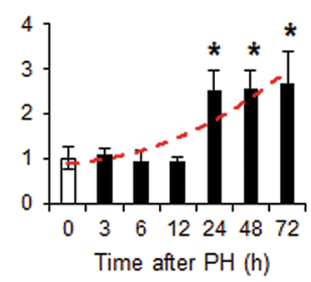

aSMA

E-cadherin

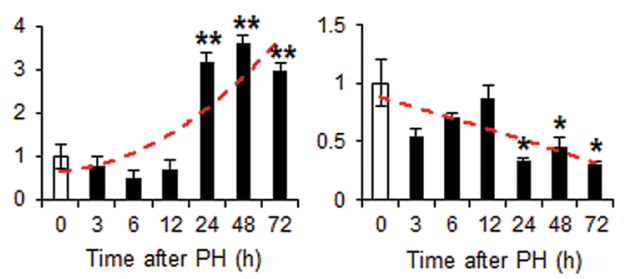

Fig. 2 Protein levels of Hh activators and EMT-related genes in hepatocytes from PH livers a Western blot analysis for SMO (86 kDa), nuclear GLI3 (145 kDa) and p65 (65 kDa), processed form of TGFb (25 kDa), VIMENTIN (57 kDa), aSMA (42 kDa), E-cadherin (120 kDa), GAPDH (36 kDa), and LAMINb1 (68 kDa) in primary hepatocytes isolated from PH livers of mice. GAPDH and LAMINb1 was used as an internal control. Immunoblots shown represent one of three independent experiments with similar results. b Cumulative densitometric analyses of SMO, GLI3, p65, TGFb, Vimentin, aSMA, and E-cadherin western blots are displayed as the mean \pm s.e.m. (unpaired two-sample Student's $t$ test, ${ }^{*} p<0.05{ }^{* *} p<0.005$ vs. 0 h) 


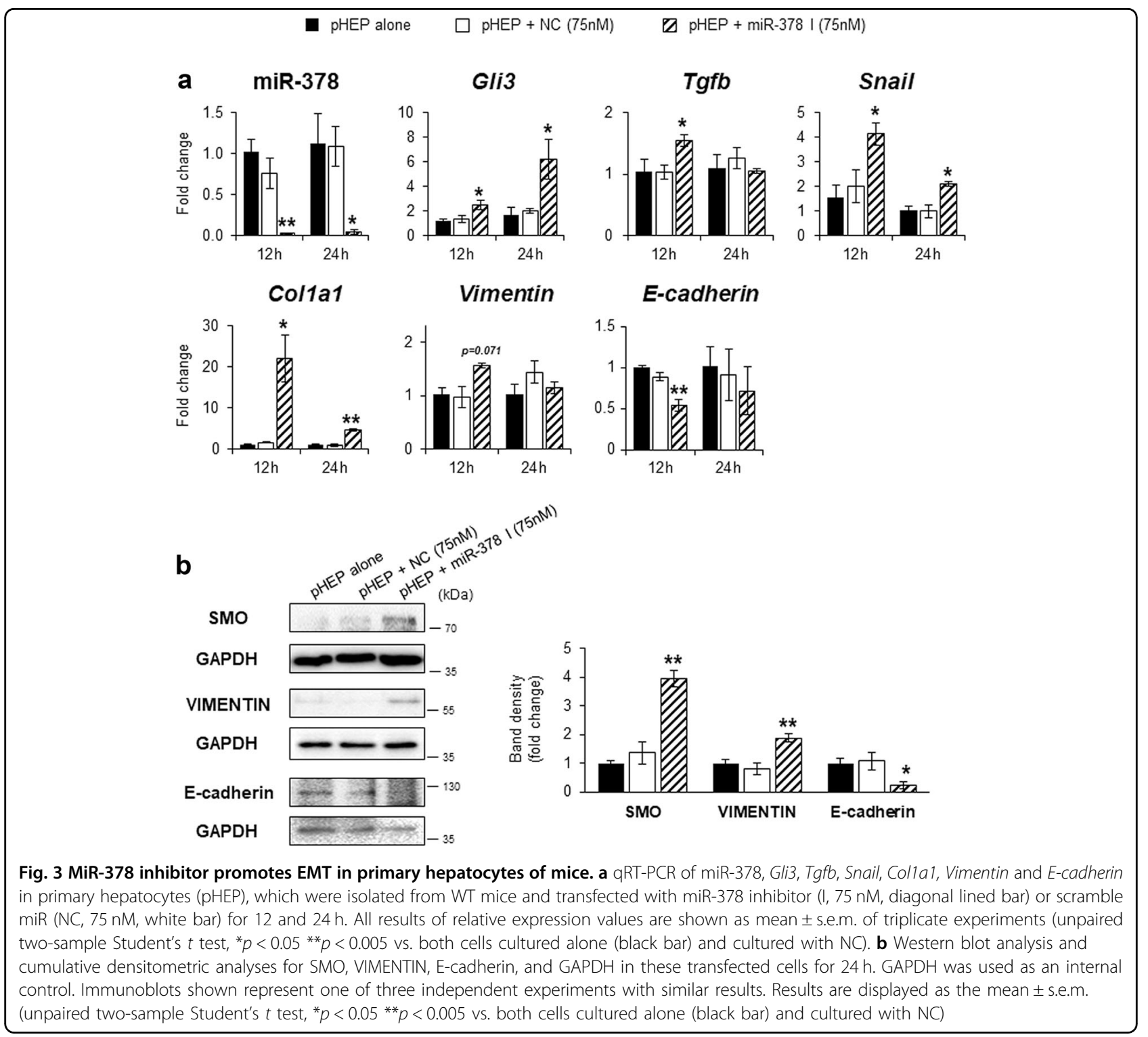

of p65 in nuclear form was low before $\mathrm{PH}$ but raised $3 \mathrm{~h}$ after $\mathrm{PH}$, and gradually declined at $12 \mathrm{~h}$. Thereafter p65 sharply increased at $48-72 \mathrm{~h}$, the time when SMO expression is maximal. The protein expression of TGFb, aSMA, and VIMENTIN, also showed the plateau after presenting the significant increase at $24 \mathrm{~h}$. E-cadherin gradually decreased after $\mathrm{PH}$, and rarely detected post 24 $h$, showing the opposite expressional pattern with the expression of mesenchymal markers (Fig. 2a, b). In addition, hepatocytes from $\mathrm{PH}$ liver at 48 and $72 \mathrm{~h}$ had the remarkably increased migratory capability compared with cells from nonPH liver, as assessed by wound-healing assay (Supplementary Figure S4). The results suggested that hepatocytes undergo EMT after PH, and Hh activators and miR-378 are associated with this process during liver regeneration.

\section{MiR-378 suppressed hepatocyte EMT}

We found that hepatocytes underwent EMT and the higher level of miR-378 in quiescent hepatocytes was significantly downregulated in hepatocytes undergoing EMT. Hence, it is possible that miR-378 prevents hepatocytes from undergoing EMT. To verify this hypothesis, we suppressed miR-378 in quiescent hepatocytes and assessed the expression of EMT-related genes in these cells. AML12, a murine cell line of normal hepatocytes, was transfected with miR-378 inhibitor or scrambled miR as negative control (NC). Compared to miR-378 expression in AML12 cells treated with or without the NC, its level was successfully reduced, followed by elevated expression of Gli3 in AML12 cells with miR-378 inhibitor at 12 or $24 \mathrm{~h}$. Levels of mesenchymal markers, Tgfb, Snail, Vimentin and Col1a1 

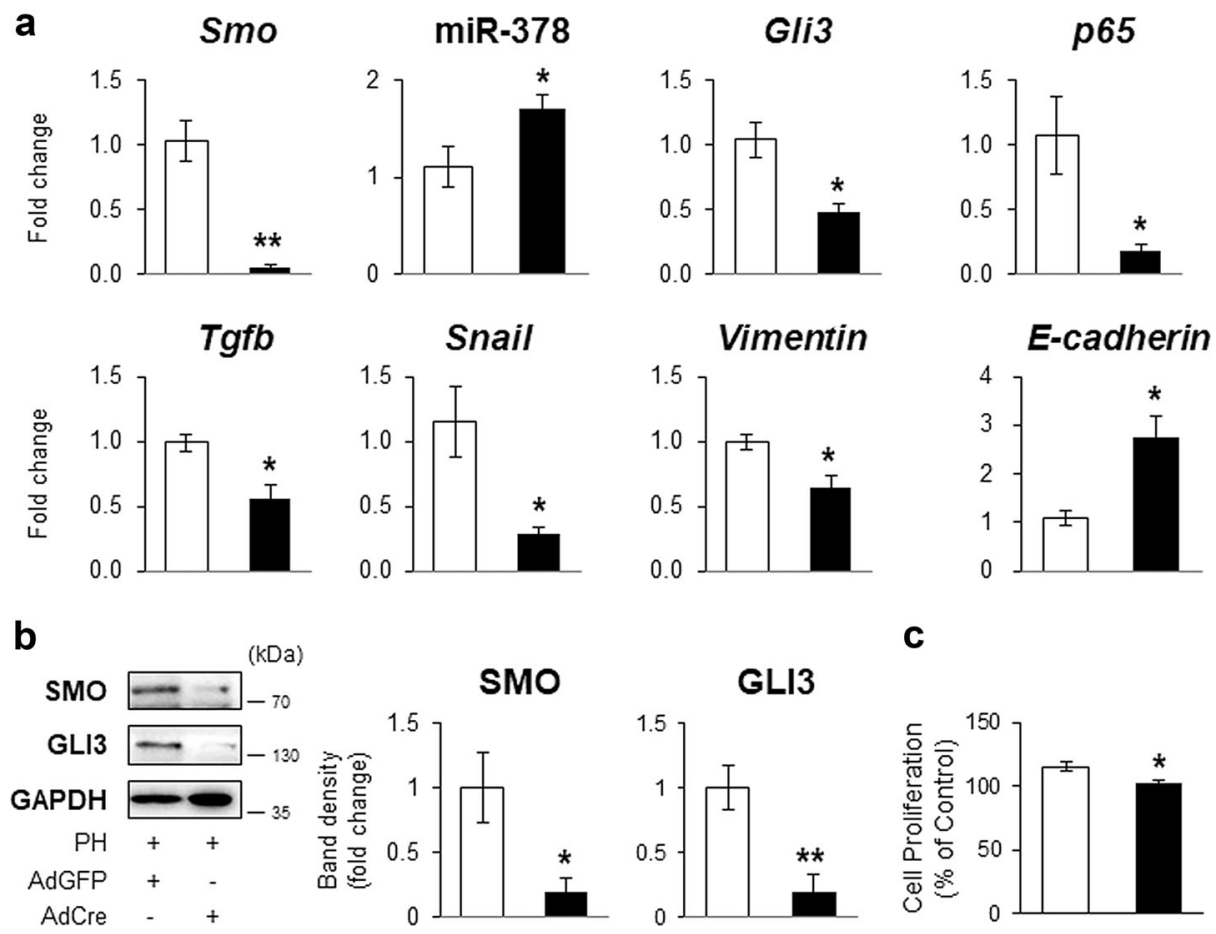

C

Fig. 4 Abrogating Hh signaling in hepatocytes disrupts EMT in hepatocytes. a qRT-PCR for Smo, miR-378, Gli3, p65, Tgfb, Snail, Vimentin, and Ecadherin in primary hepatocytes. These cells were isolated from Smo-flox transgenic mice with PH (Smo-flox with PH) at $48 \mathrm{~h}$, and then transfected with adenoviruses containing GFP (AdGFP: white bar) or Cre recombinases (AdCre: black bar) for $24 \mathrm{~h}$. All results of relative expression values are shown as mean \pm s.e.m. of triplicate experiments (unpaired two-sample Student's $t$ test, ${ }^{*} p<0.05,{ }^{* *} p<0.005$ vs. Smo-flox with PH + AdGFP). b Western blot and cumulative densitometric analysis for SMO and active form of GLI3 in these cells. GAPDH was used as an internal control. Representative images from triplicated experiments with similar results were shown. Results are displayed as the mean \pm s.e.m. (unpaired two-sample Student's $t$ test, ${ }^{*} p<0.05,{ }^{* *} p<0.005$ vs. Smo-flox with PH + AdGFP). c Cell proliferation was measured by MTS assays in these cells. The mean \pm s.e.m. results obtained from three independent experiments are graphed (unpaired two-sample Student's $t$ test, ${ }^{*} p<0.05,{ }^{* *} p<0.005 \mathrm{vs}$. Smo-flox with PH + AdGFP)

were significantly elevated in miR-378 inhibitor-treated AML12 cells, whereas the levels of epithelial markers, including E-cadherin and Zo-1 were significantly alleviated in these cell, compared with two control groups (Supplementary Figure S5).

Since primary cells better reflect the physiological state of cells in vivo compared to cell lines, we isolated hepatocytes primarily from livers of WT mice and transfected them with miR-378 inhibitor or scramble miR. The mRNA levels of Gli3, Tgfb, Snail, Col1a1, and Vimentin were upregulated following miR-378 reduction in miR378 inhibitor-treated hepatocytes, compared to primary hepatocytes treated with or without the NC (Fig. 3a). The mRNA levels of E-cadherin were downregulated in miR-378 inhibitor-treated hepatocytes, compared to other groups. Protein data also confirmed the RNA data by presenting higher expression of SMO and VIMENTIN and lower expression of E-cadherin in miR-378 suppressed hepatocytes than the other groups (Fig. 3b). These data demonstrate that miR-378 is involved in hepatocyte EMT by suppressing Gli3 expression.

\section{Smo plays the major role in the miR-378-mediated EMT in hepatocytes}

Hepatocytes undergo EMT in both the specific culture conditions and in damaged human and murine livers ${ }^{9,21,28,31-33}$. Hh signaling plays an important role in EMT $^{12,16,34}$. Our data also indicate that miR-378 is associated with EMT in hepatocytes of PH livers. Given that miR-378 was shown to be regulated by $\mathrm{SMO}^{22}$, we investigated whether Smo-mediated miR-378 was involved in hepatocyte EMT. Because Smo expression began to be upregulated significantly in hepatocytes of $\mathrm{PH}$ liver at $48 \mathrm{~h}$, hepatocytes were isolated from $\mathrm{Smo}^{\mathrm{tm} 2 \mathrm{Amc}} / \mathrm{J}$ (Smo-flox) mice at $48 \mathrm{~h}$ after $\mathrm{PH}$. And then, we conditionally depleted the Smo allele by transducing adenoviruses harboring Cre recombinase (AdCre). Adenoviruses carrying the GFP gene (AdGFP) were employed as the transfection 

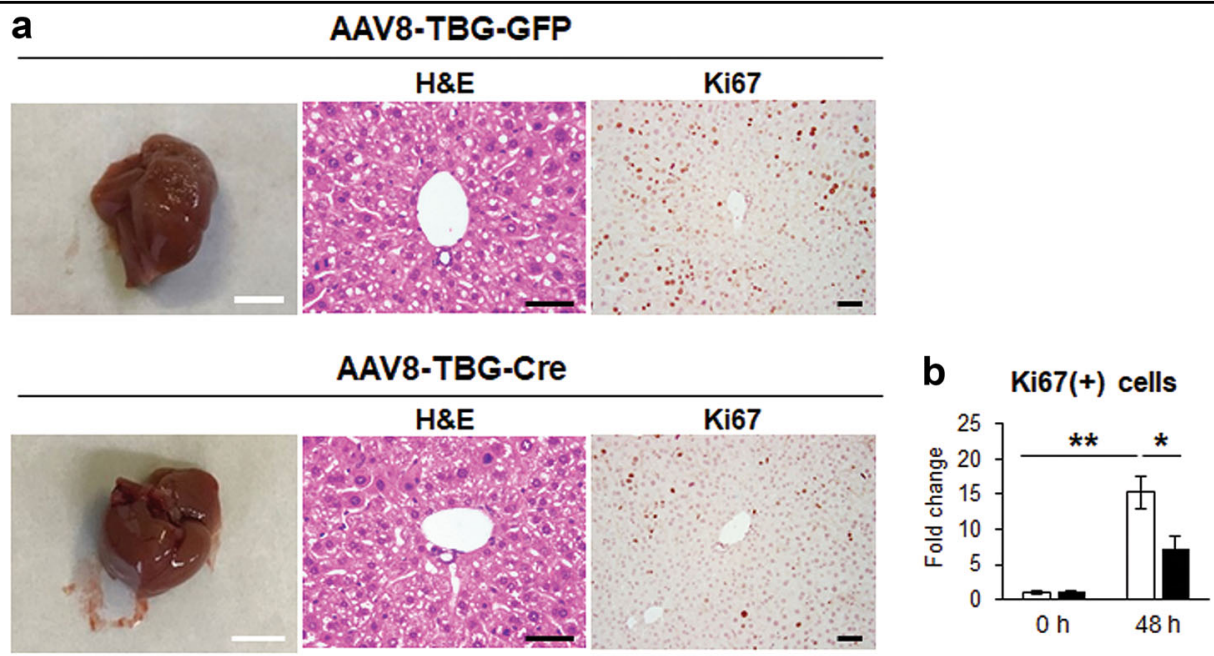

C AAV8-TBG-GFP

AAV8-TBG-Cre
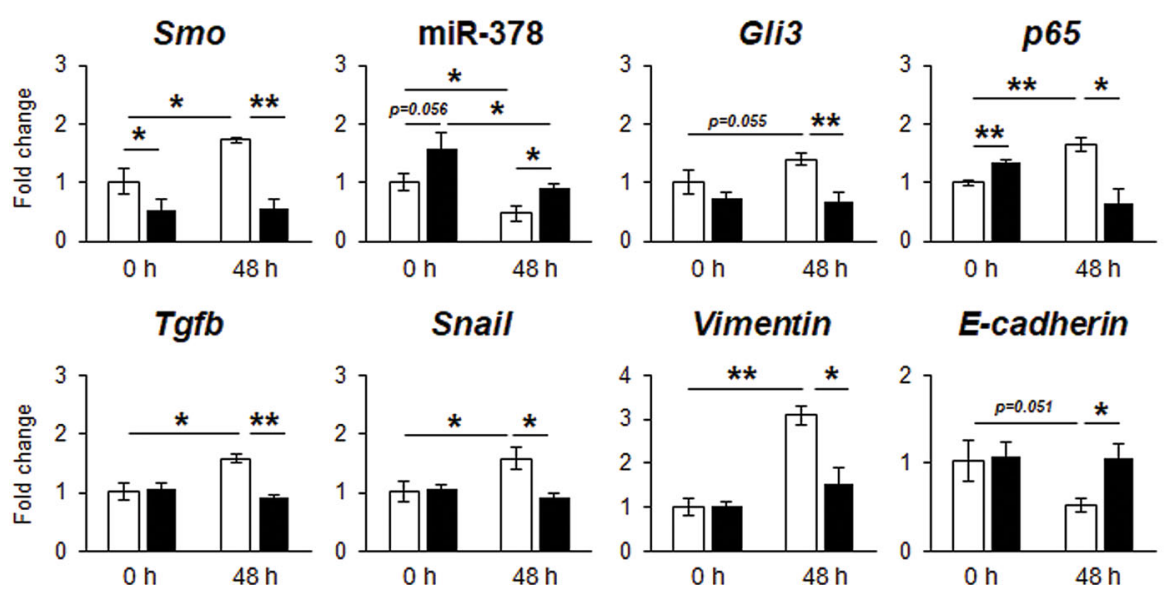

Fig. 5 Targeted deletion of Smo in livers inhibits hepatocyte proliferation and EMT after PH. a Images, $\mathrm{H}$ \& E-, and Ki67-stained sections of livers from AAV8-TBG-GFP or AAV8-TBG-Cre-treated mice at $48 \mathrm{~h}$ after PH ( $\mathrm{N}=4$ per group). Representative images of PH liver at $48 \mathrm{~h}$ were shown (liver image: scale bar $=50 \mathrm{~mm} / \mathrm{H} \& \mathrm{E}$ and Ki67 stain: scale bar $=50 \mu \mathrm{m}$ ). $\mathbf{b}$ Quantitative Ki67-stained data from these mice. Ki67-positive hepatocytic cells were quantified by counting the total number of Ki67-positive cells per field. Mean \pm s.e.m. results are graphed (one-way ANOVA with Tukey corrections, ${ }^{*} p<0.05{ }^{* *} p<0.005$ ) (c) qRT-PCR for Smo, miR-378, Gli3, p65, Tgfb, Snail, Vimentin and E-cadherin in these livers. All results of relative expression values are shown as mean \pm s.e.m. of triplicate experiments (one-way ANOVA with Tukey corrections, ${ }^{*} p<0.05{ }^{* *} p<0.005$ )

control. Hepatocytes isolated from quiescent liver also were transfected with AdCre or AdGFP, to assess the effect of adenovirus transfection in quiescent liver. The RNA levels of Smo, miR-378, Gli3, and EMT-markers, were similar between these two groups of cells from quiescent liver (Supplementary Figure S6A). Cell proliferation was not different between these two groups. However, it was significantly elevated in the AdGFPtransfected hepatocytes from $\mathrm{PH}$ livers compared with the adenovirus-transfected hepatocytes from quiescent livers (Supplementary Figure S6B). After confirming that the transfection hardly impacted on the expression of these genes in hepatocytes from quiescent livers, we compared the gene expression in adenovirus-transfected hepatocytes from PH livers of Smo-flox mice. EMT-favorable genes, including Smo, Gli3, p65, Tgfb, Snail, and Vimentin were significantly downregulated, whereas the EMT-inhibiting genes, miR-378 and E-cadherin, were significantly upregulated in AdCre-treated hepatocytes from Smo-flox mice with PH (black bar), compared to the AdGFP-treated hepatocytes from Smo-flox mice with $\mathrm{PH}$ (white bar) (Fig. 4a). The protein levels of SMO and GLI3 supported the RNA expression data, with a significant increase in SMO and GLI3 in Smo-expressing hepatocytes compared to Smo-deleted cells from $\mathrm{PH}$ livers (Fig. 4b). Smosuppressed hepatocytes also showed the significantly 


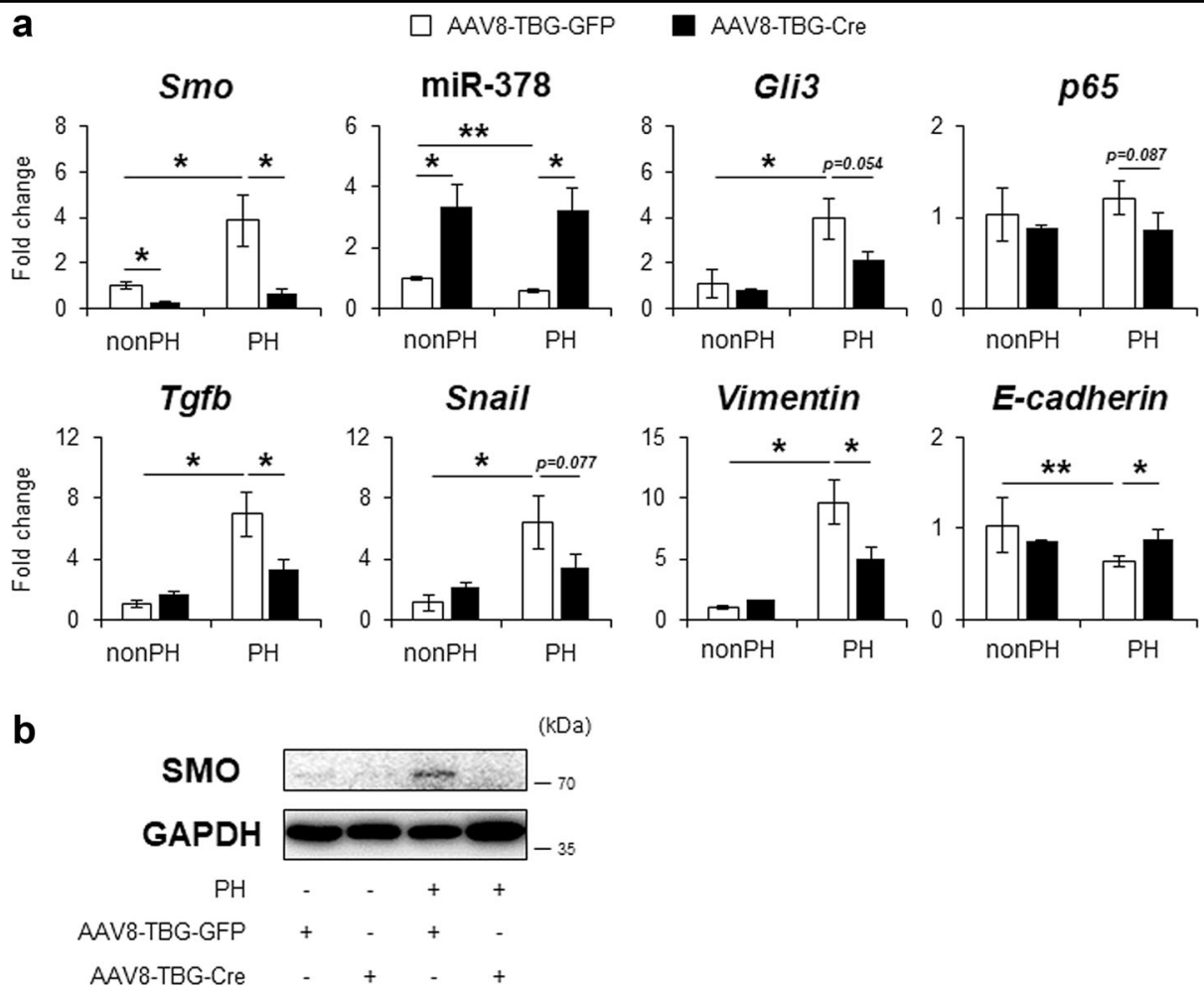

Fig. 6 Targeted disruption of Smo in hepatocytes suppressed EMT after PH. a qRT-PCR for Smo, miR-378, Gli3, p65, Tgfb, Snail, Vimentin and Ecadherin in primary hepatocytes, which were isolated from AAV8-TBG-GFP or AAV8-TBG-Cre-treated mice with (PH) or without PH (nonPH) (N=3 per group). Cells from PH livers were obtained from livers at $48 \mathrm{~h}$ post PH. All results of relative expression values are shown as mean \pm s.e.m. of triplicate experiments (one-way ANOVA with Tukey corrections, $\left.{ }^{*} p<0.05 * * 0.005\right)$. b Western blot analysis for SMO (86 kDa) and GAPDH (36 kDa) in primary hepatocytes isolated from these mice. GAPDH was used as an internal control. Immunoblots shown represent one of three independent experiments with similar results

reduced cell proliferation compared with Smo-expressing cells (Fig. 4c).

To investigate the effect of Smo on EMT in vivo, Smoflox mice were injected with a hepatocyte-tropic AAV8 viral vector bearing Cre recombinase (AAV8-TBG-Cre) to delete Smo specifically in hepatocytes. AAV8 bearing GFP (AAV8-TBG-GFP) was used as a control vector. After viral injection, mice underwent $\mathrm{PH}$ and we examined liver response at $48 \mathrm{~h}$ post $\mathrm{PH}$. Compared with AAV8-TBGGFP-treated mice, regenerating liver remnants of AAV8TBG-Cre-treated mice were relatively small and contained less Ki67-positive cells (Fig. 5a, b), although they did not show the significant change in LW and LW/BW post PH (Supplementary Figure S7). Serum levels of ALT/ AST were higher in AAV8-TBG-Cre than the AAV8TBG-GFP mice post PH (Supplementary Figure S7). The upregulated Smo, Gli3, p65, Tgfb, Snail, and Vimentin, and the downregulated miR-378 and E-cadherin in AAV8-TBG-GFP-treated PH liver were reversed in AAV8-TBG-Cre-treated PH liver, presenting the impaired EMT in $\mathrm{PH}$ liver with Smo suppression (Fig. 5c).
To confirm whether hepatocyte-specific deletion of Smo influenced the impeded EMT in PH livers of AAV8TBG-Cre-treated mice, primary hepatocytes from these mice and were analyzed for EMT-related gene expression. Increased expressions of Smo, Gli3, p65, Tgfb, Snail, and Vimentin and decreased expressions of miR-378 and Ecadherin in hepatocytes from AAV8-TBG-GFP-treated $\mathrm{PH}$ livers were reversed in cells from AAV8-TBG-Cretreated PH livers (Fig. 6a). Interestingly, regardless of PH, Smo expression was lower and miR-378 level higher in the AAV8-TBG-Cre than the AAV8-TBG-GFP group (Figs. 5 \& 6). SMO protein was rarely detected in hepatocytes from AAV8-TBG-Cre-treated mice, supporting that Cre successfully delivered into and specifically removed Smo in hepatocytes (Fig. 6b). Taken together, these results demonstrate that SMO is a master regulator of EMT in hepatocytes by altering miR-378 expression during liver regeneration.

\section{MiR-378 hinders liver regeneration in PH liver}

To investigate the function of miR-378 on liver regeneration in vivo, miR-378 mimic or NC was injected via i.p. 
route of administration into mice, which were subjected to $\mathrm{PH}$. Because most downregulation of miR-378a, upregulation of other genes and cell proliferation were observed in both livers and hepatocytes at $72 \mathrm{~h}$ post- $\mathrm{PH}$ in our analysis, we assessed the expression of miR-378 and EMT-related genes at this time point. Images of regenerating liver remnant after $\mathrm{PH}$ clearly showed that the miR-378 mimic-treated liver was relatively small compared with NC-treated liver. Serum levels of ALT/AST and the accumulation of Ki67-positive cells greatly increased in $\mathrm{PH}$ liver, compared with quiescent liver with NC. However, these enzymatic levels were significantly higher and Ki67-expressing cells were less obvious in miR-378 mimic-treated liver than two control groups (Fig. 7a, b). The LW and LW/BW also significantly declined in miR-378 treated mice with $\mathrm{PH}$ compared with two control groups of mice. The upregulated Smo, Gli3, p65, Tgfb, Snail, and Vimentin, and the downregulated miR-378 in PH liver with $\mathrm{NC}$ were reversed in $\mathrm{PH}$ liver with miR-378 mimic, presenting the alleviation of these Hh-target and EMT-related genes, and the elevation of miR378 (Fig. 7).

To assess whether the impeded liver regeneration in miR-378 mimic-treated mice resulted from the suppressed EMT in hepatocytes, the levels of miR-378 and EMT-related genes were examined in hepatocytes isolated from these mice. In line with data of gene expression in $\mathrm{PH}$ liver transfected with miR-378 or NC, primary hepatocytes from NC-treated mice with $\mathrm{PH}$ at $72 \mathrm{~h}$ had the higher level of Smo, Gli3, p65, Tgfb, Snail, and Vimentin, and the lower level of miR-378 and E-cadherin than hepatocytes from NC-treated mice without $\mathrm{PH}$. Their expressional changes were reverse in hepatocytes from miR-378 mimic-transfected $\mathrm{PH}$ liver, displaying the downregulation of Hh-activators and EMT-promoting genes and the upregulation of miR-378 and E-cadherin compared with NC-transfected hepatocytes from $\mathrm{PH}$ livers (Fig. 8a). Protein analysis also confirmed the RNA data by presenting decreased expression of SMO, GLI3, VIMENTIN and SNAIL and increased expression of Ecadherin in hepatocytes from miR-378 mimic-treated $\mathrm{PH}$ liver compared with cells from NC-treated $\mathrm{PH}$ liver (Fig. 8b). Taken together, these results suggest that miR378 is involved in liver regeneration by regulating EMT in hepatocytes.

\section{Discussion}

Many studies have reported that hepatocytes undergo EMT and acquire migratory capacity under specific EMTinducing culture conditions ${ }^{24,26,27,30,35,36}$. A previous study also showed that hepatocytes experienced intrinsic dedifferentiation in collagen monolayer cultures with upregulation of mesenchymal markers, including $\mathrm{N}$-cadherin, Vimentin, and Col1a1, although there was no change in the expression of Snail and E-cadherin, unlike the TGFb-mediated EMT response ${ }^{37}$. Although it remains controversial whether EMT occurs in response to liver damage in vivo, growing evidence suggests hepatocyte EMT in injured human and murine livers ${ }^{21,31,32}$. Albumin-expressing hepatocytes were positive for fibroblast-specific protein 1 in carbon tetrachloride $\left(\mathrm{CCl}_{4}\right)$-induced murine liver fibrosis ${ }^{30}$. In hepatitis $\mathrm{B}$ virus-infected human livers, hepatocytes were doublepositive for transferrin, a hepatocyte marker, and for collagen or Snail ${ }^{31}$. Using the lineage tracing technique, Zeisberg et al. suggested hepatocytes-undergoing EMT as a source of MFs, leading to liver fibrosis in vivo ${ }^{30}$. Reduced liver fibrosis in $\mathrm{CCl}_{4}$-treated mice with Snaildeleted hepatocytes supports EMT in hepatocytes in vivo $^{32}$. Nuclear factor erythroid 2-related factor, an important regulator of antioxidant system, has been shown to regulate EMT in renal and pulmonary fibro$\mathrm{sis}^{38,39}$ and be essential for liver regeneration in $\mathrm{PH}$ liver ${ }^{40}$. In addition, our findings demonstrated that hepatocytes undergo EMT and the Hh signaling-mediated miR-378 is involved in this process during liver regeneration of $\mathrm{PH}$ treated mice.

Although miR-378 expression was inversely correlated with expression of Smo and Gli3 in collected liver remnants at various times during liver regeneration, downregulation of miR-378 in hepatocytes from $\mathrm{PH}$ livers was observed earlier than increase in Smo, suggesting that other factors might be involved in the miR-378 reduction. Prior work demonstrated that p65 activated by SMOsuppressed miR-378 expression in HSCs, contributing to HSC activation and liver fibrosis ${ }^{22}$. As p65 is a subunit of nuclear factor kappa $\mathrm{B}(\mathrm{NF}-\mathrm{kB})^{41}$, it is possible that NF-kB signaling activators, such as tumor necrosis factor- $\alpha$, also participate in increasing p65 expression during hepatocyte transition. Hence, we examined whether p65 induced the earlier reduction of miR-378 in hepatocytes from $\mathrm{PH}$ liver. We found that nuclear form of p65 increased in hepatocytes from PH liver at $3 \mathrm{~h}$ (Fig. 2). The RNA level of p65 was also inversely correlated with miR-378 expression in hepatocytes from PH liver at various times $(r=-0.469$, $p=0.024)$, although p65 RNA tended to be upregulated at $3 \mathrm{~h}$ and began to be raised significantly at $24 \mathrm{~h}$ (data not shown).

Hh signaling regulated EMT in epithelial liver cells, such as hepatocytes and cholangiocytes, contributing to the fibrotic repair response in patients with primary biliary cirrhosis or nonalcoholic steatohepatitis (NASH). This was also demonstrated in murine models of bile duct ligation-induced biliary fibrosis or diet-induced $\mathrm{NASH}^{13,34}$. In the present study, we showed that the expression of EMT-related genes was remarkably lower in Smo-deleted hepatocytes from $\mathrm{PH}$ mice than in Smoexpressing hepatocytes from $\mathrm{PH}$ mice, suggesting that the 
a

nonPH

NC
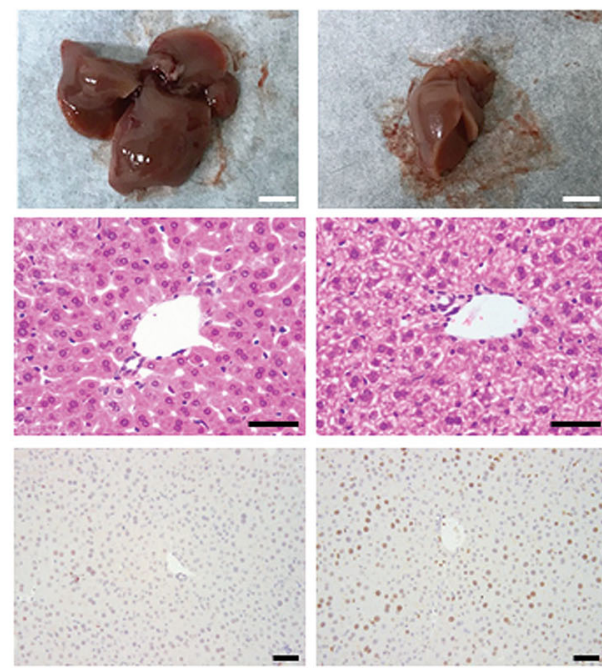

$\square$ nonPH with NC

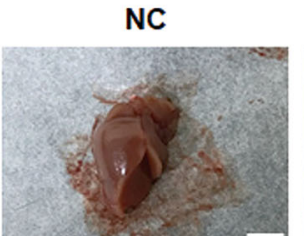

$\mathrm{PH}$ with NC

$\mathrm{PH}$

$\operatorname{miR}-378$
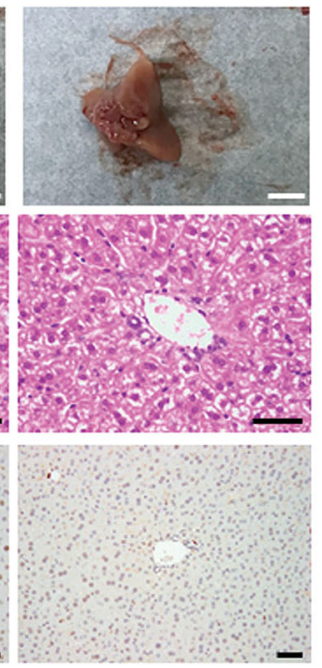

\section{Ki67(+) cells}

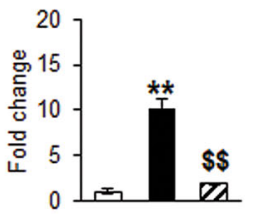

EH with miR-378

b

LW

LW/BW

ALT

AST
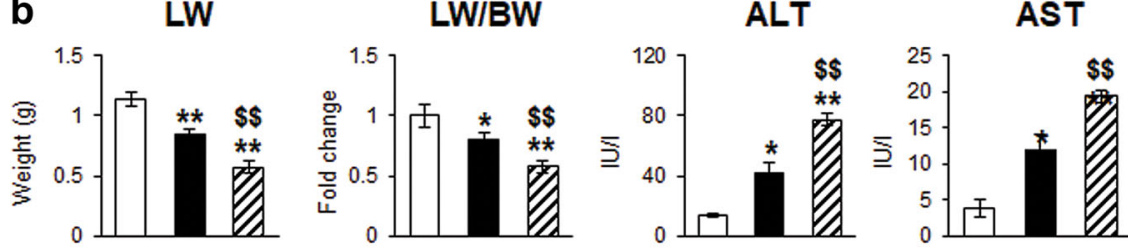

C

Smo
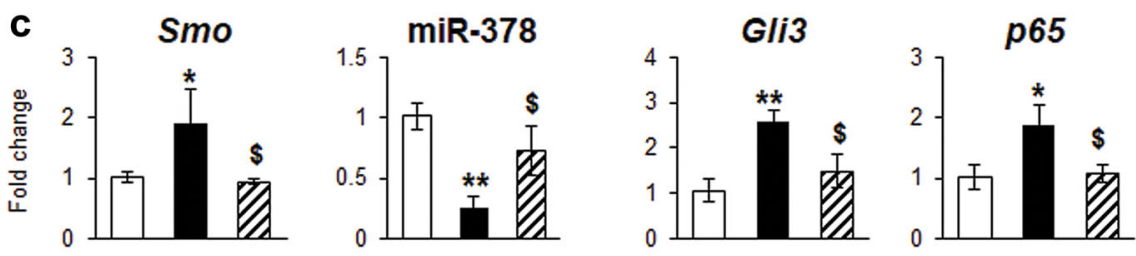

Tgfb

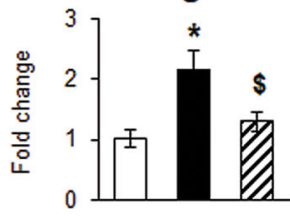

Snail

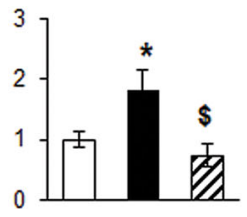

Vimentin

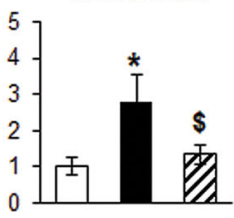

Fig. 7 Liver regeneration is impaired in miR-378 mimic-transfected mice. a Images, H \& E-, and Ki67-stained sections of livers from miR-378 mimic- or scramble RNA- (negative control: $\mathrm{NC}$ ) transfected mice with ( $\mathrm{N}=5$ per group) or without PH ( $\mathrm{N}=3$ per group). Representative images of quiescent livers (nonPH) and PH liver at $72 \mathrm{~h}$ were shown (top panel: scale bar $=50 \mathrm{~mm} /$ middle and bottom panel: $\mathrm{scale}$ bar $=50 \mu \mathrm{m}$ ). Ki67-positive hepatocytic cells were quantified by counting the total number of Ki67-positive cells per field. Mean \pm s.e.m. results are graphed (one-way ANOVA with Tukey corrections, ${ }^{*} p<0.05,{ }^{* *} p<0.005$ vs. nonPH with NC, $\$ p<0.05 \$ \$ p<0.005$ vs. PH with NC). b LW, LW/BW, and serum ALT and AST levels of each groups were graphed as mean \pm s.e.m (one-way ANOVA with Tukey corrections, ${ }^{*} p<0.05,{ }^{* *} p<0.005$ vs. nonPH with NC, $\$ p<0.05 \$ \$ p<$ 0.005 vs. PH with NC). c qRT-PCR for Smo, miR-378, Gli3, p65, Tgfb, Snail, and Vimentin in livers of these mice. All results of relative expression values are shown as mean \pm s.e.m. of triplicate experiments (one-way ANOVA with Tukey corrections, ${ }^{*} p<0.05,{ }^{* *} p<0.005$ vs. nonPH with NC, $\$ p<0.05 \$ \$ p<$ 0.005 vs. PH with NC) 


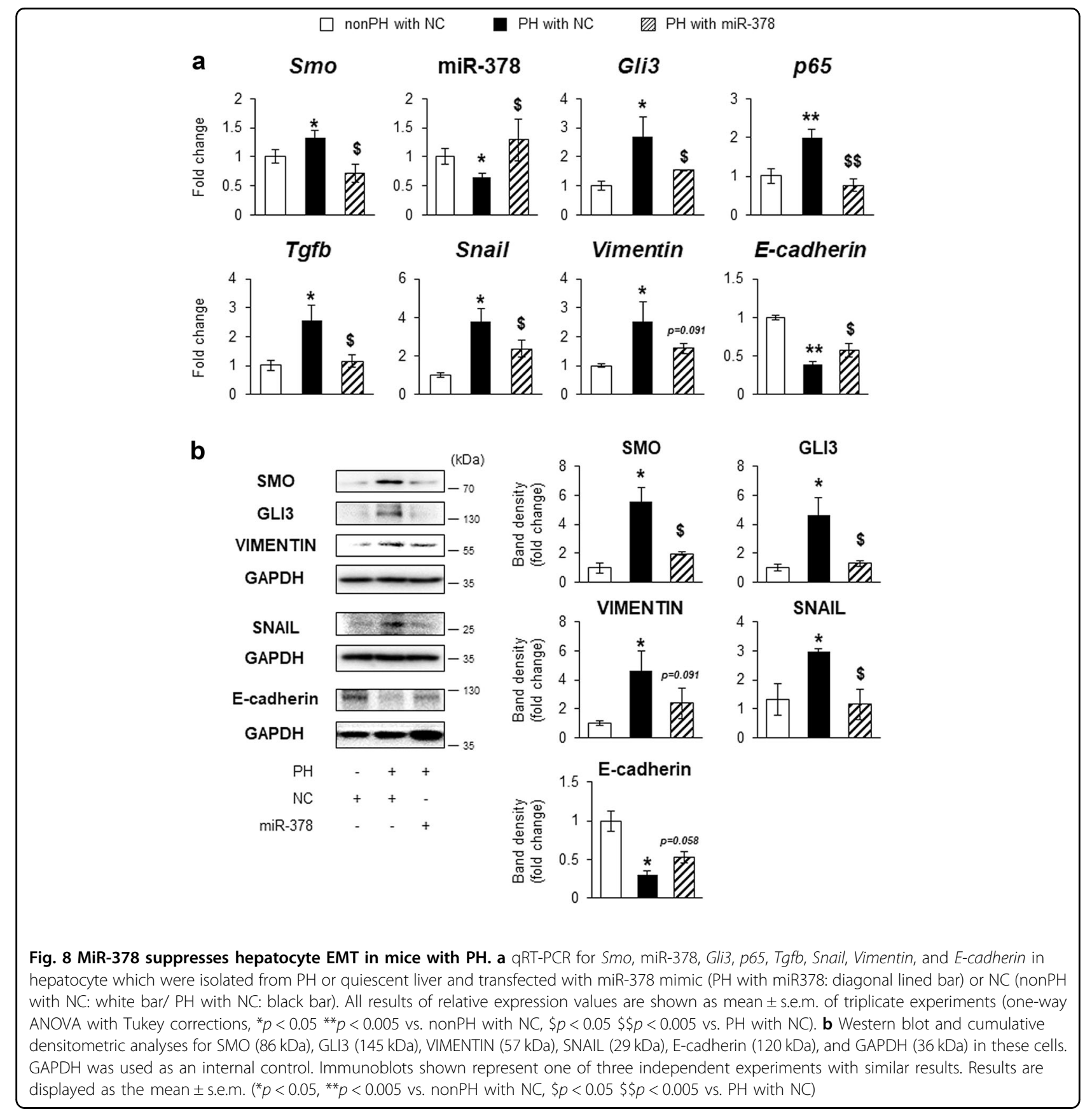

canonical Hh pathway activated through SMO plays an important role in hepatocyte EMT during liver regeneration after PH. In addition, p65 levels declined, followed by upregulation of miR-378 and downregulation of GLI3 in Smo-deleted hepatocytes (Fig. 4), indicating that SMO is an essential factor linking miR-378 with the Hh signaling pathway in promoting EMT. Hyun et $\mathrm{al}^{17}$. reported the accumulation of GLI3-positive hepatocytic cells and elevated protein levels of GLI3 with sustained fibrosis in the livers of $\mathrm{CCl}_{4}$-treated rats, whereas these were both significantly alleviated in the restored livers of rats transplanted with human chorionic plate-derived mesenchymal stem cells after $\mathrm{CCl}_{4}$ exposure. These results support that GLI3 expression influences fibrogenesis by regulating hepatocyte EMT. Therefore, our findings demonstrate that hepatocytes experience EMT during liver regeneration and miR-378 is involved in this process through interfering with the Hh pathway, particularly the SMO-GLI3 axis.

However, upregulation of EMT markers, TGFb, Snail, and Vimentin, in hepatocytes was observed earlier than increases in SMO and GLI3. Growing evidence suggest 
that the MF-HSCs impact hepatocyte reprogramming during liver regeneration ${ }^{5,10,42}$. HSCs become activated almost immediately after $\mathrm{PH}$ and these activated HSCs predominantly secret a number of factors, including $\mathrm{TGFb}^{42}$. Subsequently, TGFb induces quiescent mature hepatocyte to undergo $\mathrm{EMT}^{26,43,44}$. In line with these findings, HSC activation-related signaling factors, such as $\mathrm{TGFb}$ and Smo, were upregulated in liver at 1 and $3 \mathrm{~h}$ post $\mathrm{PH}$, respectively. Therefore, it is possible that the HSCs are first activated by TGFb and/or Hh signaling pathway, and these activated HSCs trigger hepatocyte EMT by stimulating $\mathrm{TGFb}$, which contributes to activating $\mathrm{Hh}$ signaling. In concert with TGFb signaling, the Hh signaling seems to amplify EMT in hepatocytes. However, further studies are needed to investigate the interaction of TGFb with Hh signaling in EMT of hepatocytes during liver regeneration.

\section{Materials and methods}

Experimental animals

C57BL/6 wild-type (WT) mice (Hyochang, Dae-gu, Korea) and $\mathrm{Smo}^{\mathrm{tm} 2 \mathrm{Amc}} / \mathrm{J}$ (Smo-flox) mice (Jackson Laboratory, Bar Harbor, ME, USA) were maintained in appropriate animal facilities at Pusan National University. All mice were housed with a 12-h light/dark cycle and had free access to food and water. Animal care and surgical procedures were carried out according to the provisions of the National Institutes of Health (NIH) guidelines for the Care and Use of Laboratory Animals. The animal protocol used in this study was approved by the Pusan National University Institutional Animal Care and Use Committee (PNU-IACUC) for ethical procedures and scientific care (Approval Number PNU-2016-1193).

$70 \% \mathrm{PH}$ was performed on 8-10-week-old male C57BL/6 WT mice $(N=46)$ under isofluorane anesthesia, according to the method of Higgins and Anderson ${ }^{45}$. The procedures were performed between 8 a.m. and 12 p.m. All animals resumed normal activities after recovery from anesthesia and were killed at $1 \mathrm{~h}(N=6), 3 \mathrm{~h}(N=6), 6 \mathrm{~h}(N=5), 12 \mathrm{~h}$ $(N=6), 24 \mathrm{~h}(N=6), 48 \mathrm{~h}(N=5), 72 \mathrm{~h}(N=6)$, or $96 \mathrm{~h}$ $(N=6)$ after $\mathrm{PH}$. Resected quiescent livers were used as $0 \mathrm{~h}$ comparisons and the regenerating liver remnants were formalin-fixed or snap-frozen in liquid nitrogen for subsequent analysis.

Male Smo-flox mice at 7-week-old were injected by tail vein with $1.5 \times 10^{11}$ genome copies of AAV8-TBG-GFP $(N=10)$ or AAV8-TBG-Cre $(N=10)$ (Vector Biolabs, Rockville, MD, USA) to selectively delete the Smo gene in hepatocytes. Five days after viral injection, mice underwent $\mathrm{PH}$ and were sacrificed at 48 after $\mathrm{PH}$ to obtain liver tissue or primary hepatocytes $(N=7 /$ each group). Resected quiescent livers were used as $0 \mathrm{~h}$ comparisons.
To examine the effect of miR-378 in vivo, in vivo-jetPEI (Polyplus Transfection, Illkirch, France) was used according to the manufacturer's protocols. 8-week-old male C57BL/6 WT mice were injected intraperitoneally (i.p.)with $6 \mathrm{nmol}$ per mice of miR-378 mimic $(N=5)$ or scramble miRNA $(N=5)$ (Bioneer) as a negative control $12 \mathrm{~h}$ before $\mathrm{PH}$. Mice without undergoing $\mathrm{PH}(N=3)$ were injected with scramble miRNA at the same time with $\mathrm{PH}$ group.

\section{Isolation of primary hepatocytes and cell culture}

Hepatocytes were isolated from livers of WT or Smoflox mice as described previously ${ }^{10}$. Briefly, mice were anesthetized with Zoletil50 ( $5 \mathrm{mg} \mathrm{kg}^{-1}$ body weight, Virbac S.A, France) to immobilize them in the recumbent position on a treatment table, and the inferior vena cava was cannulated under aseptic conditions. Livers were perfused in situ with EGTA and collagenase (Roche, Indianapolis, IN, USA) to disperse the cells. Primary hepatocytes were separated from nonparenchymal cells using Percoll density gradient centrifugation. As determined by Trypan Blue exclusion, cell viability was $>92 \%$ in all experiments. Primary hepatocytes were cultured on collagen-coated 6-well plates at a density of $3 \times 10^{5}$ cells per well in Williams' Medium $\mathrm{E}$ without phenol red (Sigma-Aldrich, St. Louis, MO, USA), supplemented with $5 \%$ fetal bovine serum (FBS; Gibco, Thermo Fisher Scientific, Waltham, MA, USA), $1 \mu \mathrm{M}$ dexamethasone, and a cocktail solution of penicillin/streptomycin (P/S), ITS + (insulin, transferrin, selenium complex, BSA, and linoleic acid), GlutaMAX ${ }^{\mathrm{su}}$, and HEPES (Gibco). After cell attachment ( $\sim 4-6 \mathrm{~h}$ after plating), the culture medium was replaced with serum-free Williams' Medium E containing $0.1 \mu \mathrm{M}$ dexamethasone, and a cocktail solution of $\mathrm{P} / \mathrm{S}$, ITS +, GlutaMAX ${ }^{\mathrm{sin}}$, and HEPES (Gibco) for hepatocyte maintenance.

AML12 (CRL-2254; ATCC), a cell line of normal mouse hepatocytes, was cultured on 6-well plates at a density of $3 \times 10^{5}$ cells per well in Dulbecco's Modified Eagle Medium (DMEM; Gibco) supplemented with 10\% FBS and 1\% $\mathrm{P} / \mathrm{S}$ at $37^{\circ} \mathrm{C}$ in a humidified atmosphere containing $5 \%$ $\mathrm{CO}_{2}$.

\section{Cell transfection}

To inhibit the expression of miR-378 in both primary hepatocytes and AML12, cells were transfected with $75 \mathrm{nM}$ or $100 \mathrm{nM}$ of miR-378 inhibitor (AccuTarget ${ }^{\mathrm{Tm}}$ mouse miRNA-378a-3p inhibitor, Bioneer), respectively, using Lipofectamine RNAi/MAX transfection reagent (Invitrogen, Thermo Fisher Scientific) according to the manufacturer's instructions. Equal concentrations of scrambled miRNA (miRNA inhibitor negative control \#1, Bioneer) were used as negative controls. After $12 \mathrm{~h}$, the medium was 
replaced with fresh medium, and the transfected cells were incubated at $37^{\circ} \mathrm{C}$ in a $5 \% \mathrm{CO}_{2}$ atmosphere for 12 or $24 \mathrm{~h}$.

For adenoviral transduction of primary hepatocytes isolated from Smo-flox mice with or without surgical liver resection ( $48 \mathrm{~h}$ post- $\mathrm{PH}$ ), cells were cultured overnight in serum-depleted medium before treatment. The adenoviruses harboring either the GFP gene (AdGFP; Vector Biolabs) or the Cre recombinase gene (AdCre; Vector Biolabs) were added to primary hepatocytes at a multiplicity of infection (MOI) of 80, as described previously ${ }^{46}$. Virus-containing medium was aspirated and replaced with fresh medium at $24 \mathrm{~h}$ post-transfection. The efficiency of adenovirus infection was determined by $>95 \%$ GFP-positive cells after transfection (data not shown).

\section{RNA analysis}

Total RNA was extracted from liver tissues or cells with TRIzol reagent (Ambion, Thermo Fisher Scientific). The concentration and purity of RNA were determined using a nanodrop. Template cDNA was synthesized from total RNA using the SuperScript First-strand Synthesis System (Invitrogen) or miScript Reverse Transcriptase Kit (Qiagen, Valencia, CA, USA) according to the manufacturer's protocols. We performed the real-time quantitative reverse transcriptional polymerase chain reaction (qRTPCR) analysis by using the Power SYBR Green Master Mix (Applied Biosystems, Thermo Fisher Scientific) or the miScript SYBR Green PCR Kit (Qiagen) according to the manufacturer's specifications (Eppendorf, Mastercycler Real-Time PCR). All reactions were triplicated and the data were analyzed according to the $\Delta \Delta \mathrm{C}_{\mathrm{t}}$ method. The 40 $S$ ribosomal protein S9 (RPS9) mRNA for mRNA and the U1A small nuclear RNA (RNU1A) for miRNA were used to normalize expression levels. The sequences of all primers used in this study are summarized in Supplementary Table S1. All PCR products were directly sequenced for genetic confirmation (Macrogen, Seoul, Korea).

\section{Western blot}

To extract total proteins, cells were homogenized in triton lysis buffer (TLB) and centrifuged at $13000 \mathrm{rcf}$ for $15 \mathrm{~min}$. The supernatants containing whole protein extract were used in subsequent biochemical analysis. To separate the nuclear and cytosolic fractions, cells were homogenized and suspended in buffer A (10 mM HEPES, $50 \mathrm{mM} \mathrm{NaCl}, 1 \mathrm{mM}$ DTT, $0.1 \mathrm{mM}$ EDTA, $0.1 \mathrm{mM}$ PMSF) with protease inhibitors (Roche) and incubated on ice for 20 min. After adding 0.1\% NP-40, the lysates were incubated for an additional $20 \mathrm{~min}$. After centrifugation at $5000 \times g$ for $2 \mathrm{~min}$, the supernatant was collected for the cytosolic fraction. The pellets were resuspended with buffer B (20 mM HEPES, $400 \mathrm{mM} \mathrm{NaCl}, 1 \mathrm{mM}$ DTT, 1 mM EDTA, 1 mM PMSF, 1 mM EGTA) and incubated on ice for $30 \mathrm{~min}$. After centrifugation at 13,000 r.p.m. for 15 min, the supernatant was saved for the nuclear fraction. The supernatants containing cytosolic and nuclear protein extracts were used in subsequent biochemical analyses. Protein concentration was measured with a Pierce BCA Protein Assay Kit (Thermo Scientific). Equal amounts of protein lysates were separated by 8 or $10 \%$ SDS-PAGE and then transferred onto polyvinylidene difluoride (PVDF) membranes (Millipore, Darmstadt, Germany). Rabbit anti-Smo antibody (diluted 1:1000; Abcam), rabbit anti-Gli3 antibody (diluted 1:1000; Abcam), rabbit anti-p65 antibody (diluted 1:2000; Santacruz) rabbit anti-Vimentin antibody (diluted 1:1000; Santacruz), mouse anti-aSMA antibody (diluted 1:1000; Abcam), rabbit anti-TGFb antibody (diluted 1:1000; Cell Signaling technology), mouse anti-E-cadherin (diluted 1:500; BD bioscience), rabbit anti-Snail (diluted 1:1000; Santacruz) were used as the primary antibodies. Mouse anti-GAPDH antibody (diluted 1:1000; AbD Serotec., Oxford, UK) and rabbit-anti-Lamin $\beta 1$ antibody (diluted 1:1000; Abcam) were used as internal controls for cytosolic and nuclear proteins, respectively. Horseradish peroxidase (HRP)-conjugated anti-rabbit or anti-mouse IgG (Amersham ECL ${ }^{\mathrm{mm}}$, GE Healthcare, Milwaukee, WI, USA) was used as the secondary antibody. Protein bands were detected using an EzWestLumi ECL solution (ATTO Corporation, Tokyo, Japan) per the manufacturer's specifications (ATTO Corporation, Ez-Capture II). Proteinband density was measured using CS Analyzer software (Version 3.00.1011, ATTO \& Rise Corporation). Band density of each target protein was normalized to the density of the own loading control.

\section{Liver histology and immunohistochemistry}

Liver specimens were fixed in 10\% neutral buffered formalin (NBF; Sigma), embedded in paraffin and cut into $4 \mu \mathrm{m}$ sections. The specimens were deparaffinized, hydrated, and stained with H\&E to examine hepatic morphology.

For immunohistochemistry, liver sections were deparaffinized, hydrated, and incubated in 3\% hydrogen peroxide to block endogenous peroxidase. Antigen retrieval was performed by heating in $10 \mathrm{mM}$ sodium citrate buffer (pH 6.0) for $10 \mathrm{~min}$ in a microwave. The specimens were then blocked in Protein Block solution (Dako, Carpinteria, CA, USA) for $30 \mathrm{~min}$ at room temperature (RT) followed by incubation with primary antibody at $4{ }^{\circ} \mathrm{C}$ overnight. Other sections were also incubated at $4{ }^{\circ} \mathrm{C}$ overnight in non-immune sera. Mouse Ki67 antibody (diluted 1:2000; Novocastra, Leica Microsystems, Newcastle upon Tyne, UK) was used as a primary antibody and diluted in Protein Diluent (Dako). Polymer-HRP anti-rabbit (Dako) was used as a secondary antibody and 3,3'-diaminobenzidine (DAB) for brown color was used to visualize the protein. To quantify Ki67-positive hepatocytic cells, 10 randomly 
chosen $20 \times$ fields/section were evaluated by counting the total number of Ki67-stained cells/field for each mouse.

\section{Measurement of ALT and AST}

Serum alanine transaminase (ALT/GPT, glutamatepyruvate transaminase) and aspartate transaminase (AST/GOT, glutamate-oxaloacetate transaminase) levels were measured using GOT and GPT reagents (ASAN PHARMACEUTICAL, Seoul, Korea) according to the manufacturer's instructions.

\section{Cell proliferation (MTS) assay and scratch assay}

Cell proliferation was measured with a CellTiter Proliferation Assay (Promega, Madison, WI, USA) according to the manufacturer's protocols. Briefly, cells were plated at a density of $1 \times 10^{4}$ cells per well in 96-well plates with the indicated treatment and time. After adding the MTS reagent, the plates were incubated in a $\mathrm{CO}_{2}$ incubator at $37^{\circ}$ until the color developed. Absorbance was then measured at a wavelength of $490 \mathrm{~nm}$ using a Glomax multi-detection system (Promega). Scratch assays were performed by growing cells to a confluent monolayer, and making a manual scratch using a $200 \mu \mathrm{L}$ pipette tip. Then, floating cells were washed out and fresh medium was added. As the incubation time passed, the width of scratch was narrow and it was recorded by taking photographs $(\times 20)$ using an Olympus inverted microscope (Olympus Optical Co., Ltd., Tokyo, Japan) from 24, 36 and $48 \mathrm{~h}$ after the scratch. Empty area in each time point was quantified with NIH image J version 1.49 analysis software (Rasband, W.S., ImageJ, U.S. National Institutes of Health, Bethesda, Maryland, USA, http://imagej.nih.gov/ij/, 1997-2012) and compared with that in the initiation of cell migration.

\section{Statistical analysis}

Results are expressed as mean \pm s.e.m. Statistically significant differences between the control and treatment groups or subgroups were analyzed with two-sample Student's $t$ test or one-way analysis of variance (ANOVA) followed by post hoc Tukey's test. Differences were considered significant when $p$-values were $<0.05$. The degree of correlation between the expression levels of miR-378a$3 p$ and Smo, Gli3, Tgfb, Snail, and Vimentin in primary hepatocytes isolated from $\mathrm{PH}$ liver was analyzed by the Pearson's correlation coefficient. Statistical analyses were performed using SPSS (version 21.0.0.0, IBM Corp., Armonk, NY, USA).

\section{Acknowledgements}

This work was supported by the National Research Foundation (NRF) of Korea funded by the Korean Government (MEST) (2016R1A2B2007922) to YJ

Conflict of interest

The authors declare that they have no conflict of interest.
Publisher's note

Springer Nature remains neutral with regard to jurisdictional claims in published maps and institutional affiliations.

Supplementary Information accompanies this paper at (https://doi.org/ 10.1038/s41419-018-0762-z).

Received: 16 January 2018 Revised: 21 May 2018 Accepted: 24 May 2018 Published online: 18 June 2018

References

1. Michalopoulos, G. K. Liver regeneration after partial hepatectomy: critical analysis of mechanistic dilemmas. Am. J. Pathol. 176, 2-13 (2010).

2. Fausto, N., Campbell, J. S. \& Riehle, K. J. Liver regeneration. Hepatology 43, S45-S53 (2006).

3. Fausto, N. \& Campbell, J. S. The role of hepatocytes and oval cells in liver regeneration and repopulation. Mech. Dev. 120, 117-130 (2003).

4. Michalopoulos, G. K. \& DeFrances, M. C. Liver regeneration. Science $\mathbf{2 7 6}, 60-66$ (1997).

5. Swiderska-Syn, M. et al. Myofibroblastic cells function as progenitors to regenerate murine livers after partial hepatectomy. Gut 63, 1333-1344 (2013).

6. Roskams, T. Liver stem cells and their implication in hepatocellular and cholangiocarcinoma. Oncogene 25, 3818-3822 (2006).

7. Choi, S. S. \& Diehl, A. M. Epithelial-to-mesenchymal transitions in the liver. Hepatology 50, 2007-2013 (2009).

8. Zeisberg, M. \& Neilson, E. G. Biomarkers for epithelial-mesenchymal transitions. J. Clin. Invest. 119, 1429-1437 (2009).

9. Pinzani, M. Epithelial-mesenchymal transition in chronic liver disease: Fibrogenesis or escape from death? J. Hepatol. 55, 459-465 (2011).

10. Swiderska-Syn, M. et al. Hedgehog regulates yes-associated protein 1 in regenerating mouse liver. Hepatology 64, 232-244 (2016).

11. Ochoa, B. et al. Hedgehog signaling is critical for normal liver regeneration after partial hepatectomy in mice. Hepatology 51, 1712-1723 (2010).

12. Chen, $X$. et al. Epithelial mesenchymal transition and hedgehog signaling activation are associated with chemoresistance and invasion of hepatoma subpopulations. J. Hepatol. 55, 838-845 (2011).

13. Omenetti, A. et al. Hedgehog signaling regulates epithelial-mesenchymal transition during biliary fibrosis in rodents and humans. J. Clin. Invest. 118, 3331-3342 (2008).

14. Choi, S. S., Omenetti, A., Syn, W. K. \& Diehl, A. M. The role of Hedgehog signaling in fibrogenic liver repair. Int. J. Biochem. Cell. Biol. 43, 238-244 (2011).

15. Sicklick, J. K. et al. Role for hedgehog signaling in hepatic stellate cell activation and viability. Lab. Invest. 85, 1368-1380 (2005).

16. Choi, S. S. et al. Hedgehog pathway activation and epithelial-to-mesenchymal transitions during myofibroblastic transformation of rat hepatic cells in culture and cirrhosis. Am. J. Physiol. Gastrointest. Liver Physiol. 297, G1093-G1106 (2009).

17. Hyun, J., Wang, S., Kim, J., Kim, G. J. \& Jung, Y. MicroRNA125b-mediated Hedgehog signaling influences liver regeneration by chorionic plate-derived mesenchymal stem cells. Sci. Rep. 5, 14135 (2015).

18. Hwang, H. \& Mendell, J. MicroRNAs in cell proliferation, cell death, and tumorigenesis. Br. J. Cancer 94, 776-780 (2006).

19. Sayed, D. \& Abdellatif, M. MicroRNAs in development and disease. Physiol. Rev. 91, 827-887 (2011).

20. Zhao, S. et al. Loss of MicroRNA-101 promotes epithelial to mesenchymal transition in hepatocytes. J. Cell. Physiol. 230, 2706-2717 (2015).

21. Brockhausen, J. et al. miR-181a mediates TGF- $\beta$-induced hepatocyte EMT and is dysregulated in cirrhosis and hepatocellular cancer. Liver. Int. 35, 240-253 (2015).

22. Hyun, J. et al. MicroRNA-378 limits activation of hepatic stellate cells and liver fibrosis by suppressing Gli3 expression. Nat. Commun. 7, 10993 (2016).

23. Peinado, H., Quintanilla, M. \& Cano, A. Transforming growth factor beta-1 induces snail transcription factor in epithelial cell lines: mechanisms for epithelial mesenchymal transitions. J. Biol. Chem. 278, 21113-21123 (2003).

24. Kaimori, A. et al. Transforming growth factor- $\beta 1$ induces an epithelial-tomesenchymal transition state in mouse hepatocytes in vitro. J. Biol. Chem. $\mathbf{2 8 2}$ 22089-22101 (2007). 
25. Reka, A. K. et al. Peroxisome proliferator-activated receptor- $\gamma$ activation inhibits tumor metastasis by antagonizing Smad3-mediated epithelial-mesenchymal transition. Mol. Cancer Ther. 9, 3221-3232 (2010).

26. Chen, Y. L. et al. Sorafenib inhibits transforming growth factor $\beta 1$-mediated epithelial-mesenchymal transition and apoptosis in mouse hepatocytes. Hepatology 53, 1708-1718 (2011).

27. Zhang, J. et al. miR-30 inhibits TGF- $\beta 1$-induced epithelial-to-mesenchymal transition in hepatocyte by targeting Snail1. Biochem. Biophys. Res. Commun. 417, 1100-1105 (2012).

28. Zucchini-Pascal, N., Peyre, L., de Sousa, G. \& Rahmani, R. Organochlorine pesticides induce epithelial to mesenchymal transition of human primary cultured hepatocytes. Food Chem. Toxicol. 50, 3963-3970 (2012).

29. Akkari, L. et al. Hepatitis C viral protein NS5A induces EMT and participates in oncogenic transformation of primary hepatocyte precursors. J. Hepatol. 57, 1021-1028 (2012).

30. Zeisberg, M. et al. Fibroblasts derive from hepatocytes in liver fibrosis via epithelial to mesenchymal transition. J. Biol. Chem. 282, 23337-23347 (2007)

31. Dooley, S. et al. Hepatocyte-specific Smad7 expression attenuates TGF$\beta$-mediated fibrogenesis and protects against liver damage. Gastroenterology 135, 642-659 (2008)

32. Rowe, R. G. et al. Hepatocyte-derived Snail1 propagates liver fibrosis progression. Mol. Cell. Biol. 31, 2392-2403 (2011).

33. Chen, Y., Wong, P. P., Sjeklocha, L., Steer, C. J. \& Sahin, M. B. Mature hepatocytes exhibit unexpected plasticity by direct dedifferentiation into liver progenitor cells in culture. Hepatology 55, 563-574 (2012).

34. Syn, W. K. et al. Hedgehog-mediated epithelial-to-mesenchymal transition and fibrogenic repair in nonalcoholic fatty liver disease. Gastroenterology 137, 1478-1488 (2009).

35. Meyer, C., Liu, Y., Kaul, A., Peipe, I. \& Dooley, S. Caveolin-1 abrogates TGF- $\beta$ mediated hepatocyte apoptosis. Cell Death Dis. 4, e466 (2013).
36. Nitta, T., Kim, J. S., Mohuczy, D. \& Behrns, K. E. Murine cirrhosis induces hepatocyte epithelial mesenchymal transition and alterations in survival signaling pathways. Hepatology 48, 909-919 (2008).

37. Meyer, C. et al. Distinct dedifferentiation processes affect caveolin-1 expression in hepatocytes. Cell. Commun. Signal. 11, 6 (2013).

38. Kanlaya, R., Khamchun, S., Kapincharanon, C. \& Thongboonkerd, V. Protective effect of epigallocatechin-3-gallate (EGCG) via Nrf2 pathway against oxalateinduced epithelial mesenchymal transition (EMT) of renal tubular cells. Sci. Rep. 6, 30233 (2016).

39. Zhou, W. et al. Nrf2 inhibits epithelial-mesenchymal transition by suppressing snail expression during pulmonary fibrosis. Sci. Rep. 6, 38646 (2016).

40. Beyer, T. A. et al. Impaired liver regeneration in Nrf2 knockout mice: role of ROS-mediated insulin/IGF-1 resistance. EMBO J. 27, 212-223 (2008).

41. Luedde, T. \& Schwabe, R. F. NF-kB in the liver-linking injury, fibrosis and hepatocellular carcinoma. Nat. Rev. Gastroenterol. Hepatol. 8, 108-118 (2011).

42. Mabuchi, A. et al. Role of hepatic stellate cell/hepatocyte interaction and activation of hepatic stellate cells in the early phase of liver regeneration in the rat. J. Hepatol. 40, 910-916 (2004).

43. Blobe, G. C., Schiemann, W. P. \& Lodish, H. F. Role of transforming growth factor beta in human disease. N. Eng. J. Med. 342, 1350-1358 (2000).

44. Gressner, A. M., Weiskirchen, R., Breitkopf, K. \& Dooley, S. Roles of TGF-beta in hepatic fibrosis. Front. Biosci. 7, d793-d807 (2002).

45. Higgins, G. M. \& Anderson, R. M. Experimental pathology of the liver. Arch. Pathol. 272, 186-202 (1931).

46. Michelotti, G. A. et al. Smoothened is a master regulator of adult liver repair. J. Clin. Invest. 123, 2380-2394 (2013). 